\title{
Computation of Steady State Nodal Voltages for Fast Security Assessment in Power Systems
}

Møller, Jakob Glarbo; Jóhannsson, Hjörtur; Østergaard, Jacob

Published in:

Proceedings of IEEE Electric Power Quality and Supply Reliability Conference 2014

Link to article, DOI:

10.1109/PQ.2014.6866783

Publication date:

2014

Document Version

Early version, also known as pre-print

Link back to DTU Orbit

Citation (APA):

Møller, J. G., Jóhannsson, H., \& Østergaard, J. (2014). Computation of Steady State Nodal Voltages for Fast Security Assessment in Power Systems. In Proceedings of IEEE Electric Power Quality and Supply Reliability Conference 2014 (pp. 51-55). IEEE. https://doi.org/10.1109/PQ.2014.6866783

\section{General rights}

Copyright and moral rights for the publications made accessible in the public portal are retained by the authors and/or other copyright owners and it is a condition of accessing publications that users recognise and abide by the legal requirements associated with these rights.

- Users may download and print one copy of any publication from the public portal for the purpose of private study or research.

- You may not further distribute the material or use it for any profit-making activity or commercial gain

- You may freely distribute the URL identifying the publication in the public portal 


\title{
Computation of Steady State Nodal Voltages for Fast Security Assessment in Power Systems
}

\author{
Jakob Glarbo Møller, Hjörtur Jóhannsson, and Jacob Østergaard
}

\begin{abstract}
Development of a method for real-time assessment of post-contingency nodal voltages is introduced. Linear network theory is applied in an algorithm that utilizes Thevenin equivalent representation of power systems as seen from every voltage-controlled node in a network. The method is evaluated by comparing with results from time domain simulations and power flow calculations using NewtonRaphson's method. It is concluded that the developed method performs better than Newton-Raphson's method in reproducing results from time domain simulations. Discussion includes considerations for further development for facilitating treatment of composite loads.
\end{abstract}

Index Terms-Power System Security, Network Theory, Linear Systems, Power System Analysis Computing.

\section{INTRODUCTION}

Tendencies in decentralized and stochastic generation implies that security assessments of power systems must embrace greater diversity and uncertainty. This will undoubtedly increase the computational burden if traditional methods are to converge with adequate confidence. On top of this a deregulation of many electricity markets is in progress with intentions of increasing power exchange from low-price zones to high-price zones. This imposes requirements of closer coordination between system operators. Experience show that management of system security across regions of responsibility is a non-trivial task and misunderstandings have resulted in blackout [1]. In light of this it is apparent that attention must be given to the continued development of operational security and stability of power systems.

Great advancements were made during the last decades in terms of obtaining and processing system measurements. Phasor measurement units (PMUs) and phasor data concentrators are being deployed worldwide [2]. Phasor data concentrators can refer local measurements in a common timeframe. This allows instantaneous snapshots of currents and voltages to be obtained for every cycle of system frequency [3].

It is a probable scenario that future transmission system operators will have such continuous streams of system wide PMU snapshots as a central mean in system assessment. Wide area measurements of currents and voltages allow models that use network admittances to be applied with greater precision in on-line assessments. This drives develop-

The presented research is part of the project on Secure Operation of Sustainable Power Systems (SOSPO). The project is funded by the Danish Strategic Research Council under grant agreement number 11-116794. For more information on the SOSPO project visit www.sospo.dk.

Authors are with Center for Electric Power and Energy at Technical University of Denmark (Contact: jglmo@elektro.dtu.dk). ment of methods as presented in [4] and [5] that assesses the stability of a present system state based on PMU snapshots.

With more frequent and coherent sets of measurements it becomes feasible to consider adaptability of assessment methods using Thevenin equivalent representation to realtime operation as discussed in [6] and [7].

Contingency assessment is an important tool in managing power system security. The fundamental principle is to test the severity of a predefined set of disturbances in order to operate the system defensively. This is necessary because disruptive events have a tendency of escalating fast and operators might not be able to apply counter measures in due time [8].

Static contingency assessment treats events that may be studied fully by their steady-state response. This will usually include loss of any element without a fault ( $\mathrm{N}-1)$.

Present day real-time implementation of static contingency assessment has been reported to handle 1000 contingencies every 3 minutes [9]. Recent and significant results in real-time contingency analysis on very large systems have been achieved by means of parallel computations [10].

Widely preferred algorithms for contingency assessment are based on Newton-Raphson's power flow method. However, compared with time domain simulations difficulties in providing precise estimates of nodal voltages by means of power flow methods might arise.

With realistic post-contingency PMU snapshots, methods as described in [4] and [5] can be used to assess distance to instability and thus allow defensive counter actions to be applied.

This paper introduces the development of a Thevenin equivalent based method that may be used in obtaining postcontingency snapshots of nodal voltages.

Section II. explains the principles of the method being developed. In section III. the method is evaluated by comparison to Newton-Raphson's method and time domain simulations. Section IV. provides a discussion of the method and means of evaluation while section $\mathrm{V}$. concludes the paper.

\section{METHOD}

Phasor representation of positive sequence voltages and currents provides a linear system representation that covers a balanced three phase AC system. The linearization applies to power transmission systems when it is sufficient to apply equivalent $\pi$ models of branch elements and loads behave as constant impedances. Such networks may be aggregated into a Thevenin's equivalent through operations treated later in this section. 


\section{A. Voltage Angles at Voltage Controlled Nodes}

We will begin by introducing the dependency between the voltage angle at a node and the power injection at the same node with the network represented by Thevenin's equivalent.

Such a system is depicted in Fig. 1 in which the Thevenin impedance may be written $Z_{t h}=\left|Z_{t h}\right| \angle \theta_{t h}=R_{t h}+\boldsymbol{i} X_{t h}$.

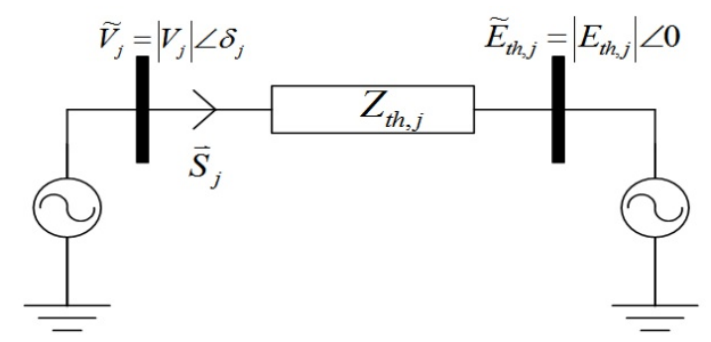

Fig. 1. Power injection $\vec{S}_{j}$ at voltage controlled node $j$ with the remaining network represented by Thevenin's equivalent.

The active power injection at node $j$ is given by:

$$
\begin{gathered}
P_{j}=\frac{X_{t h, j}\left|V_{j}\right|\left|E_{t h, j}\right|}{R_{t h, j}^{2}+X_{t h, j}^{2}} \sin \delta_{j}-\frac{R_{t h, j}\left|V_{j}\right|\left|E_{t h, j}\right|}{R_{t h, j}^{2}+X_{t h, j}^{2}} \cos \delta_{j} \\
+\frac{R_{t h, j}\left|V_{j}\right|^{2}}{R_{t h, j}^{2}+X_{t h, j}^{2}}
\end{gathered}
$$

If the voltage magnitude at node $j$ and the Thevenin equivalent are constants, (1) is of two dimensions and known as the $P-\delta$ curve (Fig. 2). The $P-\delta$ curve can be used to obtain information that is central to angle stability studies [11].

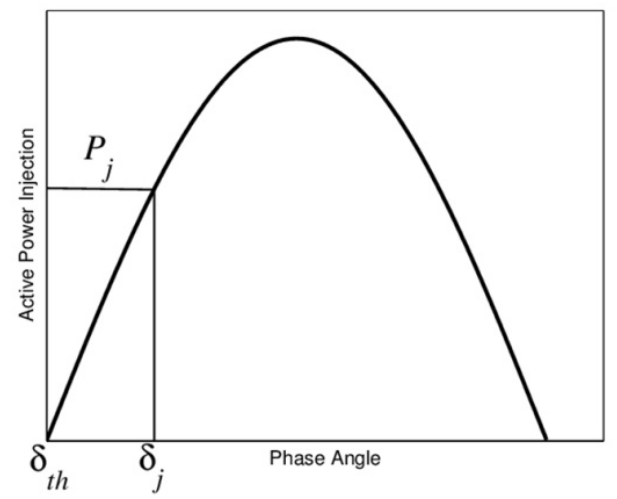

Fig. 2. $P-\delta$ curve; relation between active power injection and voltage angle at a point $j$ of constant voltage.

Rewriting (1) allows the phase angle of $\tilde{V}_{j}$ to be found on basis of active power injection:

$$
\delta_{j}=\arccos \left(\frac{P_{j}\left|Z_{t h, j}\right|^{2}-R_{t h, j}\left|V_{j}\right|^{2}}{\left|Z_{t h, j}\right|\left|V_{j}\right|\left|E_{t h, j}\right|}\right)+\theta_{t h, j}
$$

This means that the voltage angle at a node may be obtained algebraically on basis of knowledge to the voltage magnitude and active power injection at that node with the remaining network represented by its Thevenin equivalent.

\section{B. Thevenin Equivalents in Common Reference}

The network is represented by an admittance matrix ordered according to Schur's complement;

$$
\left(\begin{array}{c}
0 \\
\tilde{I}_{v c}
\end{array}\right)=\left(\begin{array}{cc}
\boldsymbol{Y}_{n c} & \boldsymbol{Y}_{\text {link }} \\
\boldsymbol{Y}_{\text {link }}^{T} & \boldsymbol{Y}_{v c}
\end{array}\right)\left(\begin{array}{c}
\tilde{V}_{n c} \\
\tilde{V}_{v c}
\end{array}\right)
$$

where $v c$ is the set of voltage controlled nodes and $n c$ is the set of non-controlled nodes. Loads are represented by admittance values and added to the diagonal elements of $\boldsymbol{Y}$.

The Thevenin equivalent impedance as experienced at a voltage controlled node $j$ may be obtained by taking the inverse of a matrix that is formed of the block admittance matrix of non-controlled nodes concatenated with those elements of $\boldsymbol{Y}_{v c}$ and $\boldsymbol{Y}_{\text {link }}$ that corresponds to the node $j$ [12].

$$
\boldsymbol{Z}^{(j)}=\left(\begin{array}{cc}
\boldsymbol{Y}_{n c} & Y_{\text {link,j }} \\
Y_{\text {link,j }}^{T} & Y_{(j, j)}
\end{array}\right)^{-1}
$$

The reduced system of (4) represents a network where all $v c$ nodes, except $j$, are short circuited and $j$ is open circuited. By definition the impedance that may be measured between $j$ and ground under this circuit representation is the Thevenin impedance $Z_{t h, j}$ seen from node $j$. This is equivalent to the last diagonal element of $\boldsymbol{Z}^{(\mathrm{j})}$ :

$$
Z_{t h, j}=Z^{(j)}(|n c|+1 ;|n c|+1)
$$

The Thevenin equivalent voltage as experienced at a node $j$ can be decomposed into factors of voltages at the nearby voltage controlled nodes;

$$
\tilde{E}_{t h, j}=\sum_{\substack{i \in v c \\ i \neq j}} k_{i, j} \tilde{V}_{i}
$$

where the factors $k_{i, j}$ were named grid transformation coefficients by [13]. These are obtained from a reduced and inverted admittance matrix. Concatenating the block admittance matrix of non-controlled nodes with elements corresponding to node $j$ for which the Thevenin voltage is to be found and any node $i$ of the remaining voltage controlled nodes gives an $|n c|+2$ by $|n c|+2$ matrix of which the inverse can be used to obtain $k_{i, j}$.

$$
\boldsymbol{Z}^{(i, j)}=\left(\begin{array}{ccc}
\boldsymbol{Y}_{n c} & Y_{\text {link,j }} & Y_{\text {link }, i} \\
Y_{\text {link,j }}^{T} & Y_{(j, j)} & Y_{(j, i)} \\
Y_{\text {link }, i}^{T} & Y_{(i, j)} & Y_{(i, i)}
\end{array}\right)^{-1}
$$

The grid transformation coefficient $k_{i, j}$ with which $\tilde{V}_{i}$ contributes to $\tilde{E}_{t h, j}$ is then found in the reduced system of (7) as a relation of the impedance between node $i$ and ground and the impedance between $i$ and $j$.

$$
k_{i, j}=\frac{\boldsymbol{Z}^{(i, j)}(|n c|+1 ;|n c|+2)}{\boldsymbol{Z}^{(i, j)}(|n c|+2 ;|n c|+2)}
$$

Complex impedance values yield complex grid transformation coefficients.

Equation (6) may be written on matrix form where $\boldsymbol{G T C}$ is a $|v c|$ by $|v c|$ matrix of grid transformation coefficients [9];

$$
\left(\tilde{E}_{t h}\right)=(\boldsymbol{G T C})\left(\tilde{V}_{v c}\right)
$$

Equation (9) allows Thevenin voltages to be obtained within a common frame of reference. This in turn allows voltage angles obtained from (2) to be referred in a common frame.

\section{Thevenin Equivalent based Static Contingency Assessment}

The developed method is a Thevenin equivalent based static contingency assessment (TESCA).

Equations (2) and (9) are central in the iterative method developed for obtaining post-contingency steady-state nodal 
voltages. The basic assumptions are constant active power injections and constant voltage magnitudes at voltage controlled nodes. Loads are represented by constant impedance and resistive transmission losses are neglected.

The only type of contingency treated in this study is loss of a single transmission line. Removal of a branch element is applied by perturbing Thevenin impedances and grid transformation coefficients throughout the network.

Iterative solution to the problem is achieved by repetition of two steps:

1. Updating the set of Thevenin voltages by solving (9) with the perturbed $\boldsymbol{G T C}$ matrix.

2. Updating voltage angles at all $v c$ nodes by solving (2) with the new Thevenin voltages and perturbed Thevenin impedance.

The procedure is repeated until a stable set of voltage angles is obtained. From voltages at the voltage controlled nodes and knowledge to the perturbed admittance matrix it is possible to obtain voltages at non-controlled nodes using:

$$
\left(\tilde{V}_{n c}\right)=-\boldsymbol{Y}_{\text {link }} \boldsymbol{Y}_{n c}^{-\mathbf{1}}\left(\tilde{V}_{v c}\right)
$$

The result is thus a full set of stable post-contingency nodal voltages reflecting a post-contingency PMU snapshot.

\section{RESULTS}

Testing of TESCA was done by comparing results of contingency cases with results obtained from time domain simulations. Taking the time domain results as reference a comparison with the Newton-Raphson's power flow method $(N R)$ was also made in order to evaluate TESCA against this widely preferred method for contingency analysis.

\section{A. Test System and Reference Case Simulations}

The test system used is inspired by the Nordic32 test system and was implemented in PSS/E [14]. The system consists of 46 nodes of which 20 are voltage controlled. Modifications were made to branch elements as to neglect resistive losses and generating units in order to represent them with identical dynamic characteristics. Excitation limiters of generating units were disabled as was tap-changing of transformers.

A contingency assessment is conducted in PSS/E with time domain simulations. The cases studied reflect the total set of 33 individual $\mathrm{N}-1$ cases related to loss of a single $400 \mathrm{kV}$ line. Time response to every contingency was studied to determine an instant of steady-state at which a snapshot of nodal voltages could be taken. This snapshot would be used as reference for comparing with the methods under test.

\section{B. Contingency Analysis by Methods Under Test}

NR simulations were conducted in PSS/E using the same modifications to the test system as described above. The input scenario was identical to that used for time domain simulations except the selection of a slack-bus at which active power mismatches are balanced as required in $N R$. The power flow method converged in all 33 scenarios.

TESCA is implemented in Matlab. Simulations are conducted on an input scenario composed of an admittance matrix and an initial set of nodal voltages and power injections. The input scenario is consistent with that used for time domain simulations to a precision of $10^{-5}$. The Thevenin impedances and $\boldsymbol{G T C}$ matrix were perturbed according to the list of 33 contingencies and post-contingency snapshots of steady-state nodal voltages were obtained.

\section{Presentation of Results}

Comparing the nodal voltages requires a common angular reference. A reference node is chosen as a solid reference between the datasets originating from the three methods. All snapshots of post contingency nodal voltages are rotated so the voltage angle at the reference node is exactly identical in all data sets. Errors between results obtained by the methods under test and the time domain reference cases is stated in terms of a total vector error (TVE) [15]:

$$
T V E=\frac{\left|\tilde{V}_{M U T}-\tilde{V}_{T D}\right|}{\left|\tilde{V}_{T D}\right|} \cdot 100 \%,
$$

where suffix $M U T$ is method under test and $T D$ is time domain result. TVE is calculated for every single voltage phasor of a snapshot.

Choice of reference node impacts the distribution of TVEs over a snapshot as any error originating from the angle of the reference phasor will be transferred to the remaining TVEs of the system. Therefore results of the methods under test are evaluated on basis of the single largest TVE in every post-contingency snapshot.

Fig. 3 shows contingency cases ordered according to descending error of $N R$ results together with the corresponding maximum error of TESCA results. The figure shows that TESCA reproduces the time domain results with significantly better precision than $N R$. Of the 33 cases studied all TESCA results are within $3.0 \%$ TVE and most are within $1.0 \% \mathrm{TVE}$

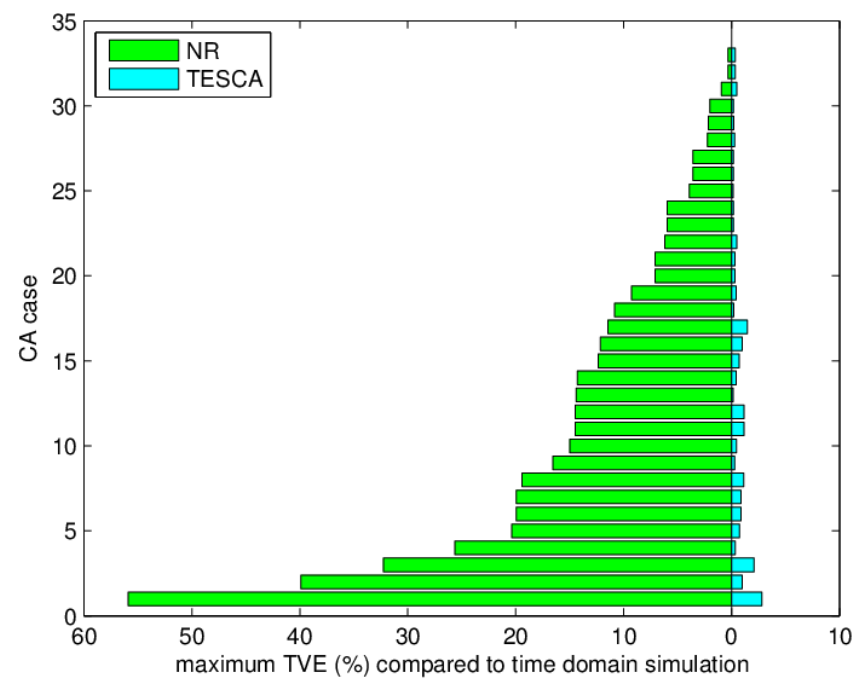

Fig. 3. The single largest vector error (\%) of a voltage phasor obtained from approximate methods in each case in the contingency assessment.

The reason for the poor performance of $N R$ in the problem of reproducing nodal voltages is that this is not the purpose of $N R$. $N R$ solves the power injection equations and a large error could arise from differences in power injection at the slack bus. In Fig. 4 the methods under test are evaluated in terms of summed residual squares of active power injection. The general picture is that the two methods perform somewhat similar with a slight advantage to TESCA.

A closer look at the active power injections reveals that TESCA usually but not always arrives at a power injection that is on the higher side of time domain results. This means 
that there is no guarantee for conservative results in terms of machine loadings with this implementation of TESCA.

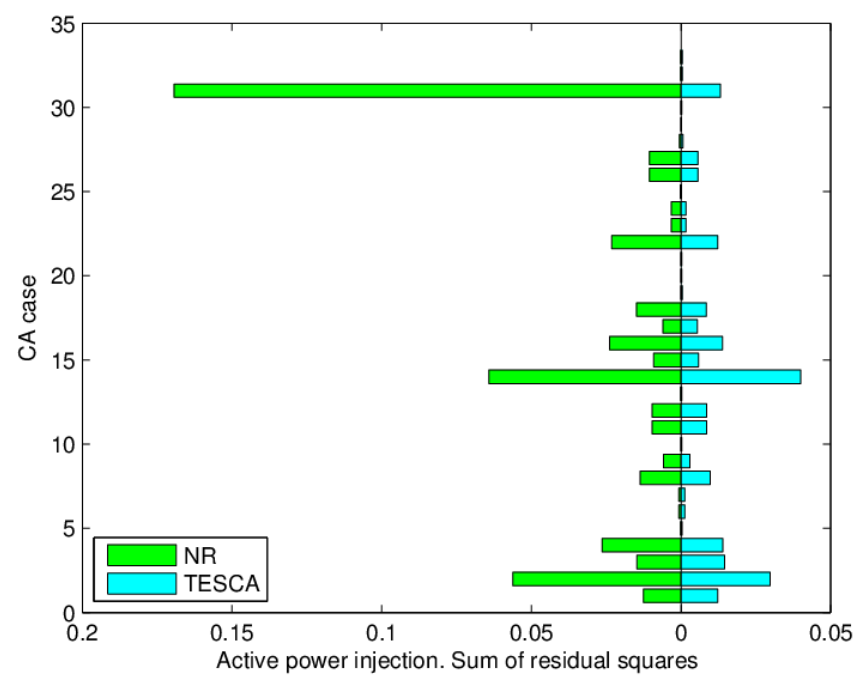

Fig. 4. Sum of residual squares of active power injection obtained from approximate methods compared to time domain simulation.

\section{Runtime performance of TESCA}

The number of iterations needed for TESCA to converge is substantial. Fig. 5 shows the iteration count on every case in the study.

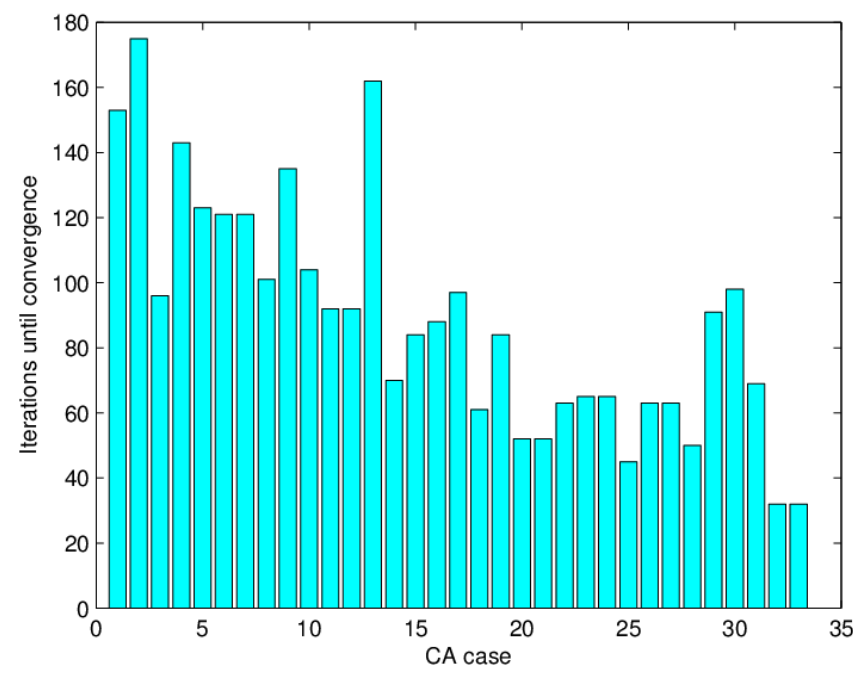

Fig. 5. Number of iterations until convergence of TESCA contingency studies.

Despite a large number of iterations the wall-clock time spend until TESCA converges is quite low. This is due to the lightness of the problems being solved during the iterative process. Equation (9) is a forward system of linear equations and (2) is a set of $|v c|$ independent algebraic equations both relatively light computational problems.

On one thread of an Intel Core i7 CPU at $2.4 \mathrm{GHz}$ the total time spend solving the full set of 33 contingencies as presented above was $1.43 \mathrm{~s}$. Profiler results for the Matlab code reveals that $90 \%$ of the runtime when solving contingencies with TESCA is spend on inversion of the admittance matrices that are used for finding Thevenin impedances and grid transformation coefficients.

\section{DISCUSSION}

A simple study shows that the developed method outperforms Newton-Raphson's method in reproducing post contingency nodal voltages if a reference case is obtained from time domain simulations. This conclusion is based on a study that relies on a chain of assumptions regarding system behavior which to some extend must be considered farfetched. Neglecting over excitation limits is equivalent of assuming infinite reactive power reserves which leads to a non-conservative and imprecise generation system model. This assumption was made to provide constant voltage magnitudes as boundary values for the developed method. If the method is to be used for security assessment it should at least be accompanied by checking of limit violations of reactive power injection.

Load representation by constant impedance is a drastic simplification of system response. To facilitate composite load handling will require some form of iterative recalculation of the Thevenin equivalents used by the developed method. Load representation deserves particular attention in further development and dynamic models must be addressed to facilitate under load tap-changing transformers.

Differences in participation in frequency control of generating units will impact the post-contingency steady state equilibrium and thereby the post-contingency PMU snapshot. The effect of assuming identical dynamic characteristics of generating units on precision of results must be investigated.

It was noted that the present implementation of the developed method does not provide conservative results in terms of machine loadings. It may be possible to resolve this issue by introducing composite loads. However, reduced conservativeness is likely to be a necessary trade off when aiming for high precision.

The computational performance of the presented method is severely reduced by the number of matrix inversions needed to find the grid transformation coefficient and Thevenin impedances in every contingency case. It is expected that optimization of this step can be achieved through application of inverse matrix modification schemes [16].

The current implementation of the presented method assesses naively the grid transformation coefficient between all $|v c|^{2}$ pairs of voltage controlled nodes. It is expected that further optimization of the computational performance is achieved through application of a scheme that only calculates grid transformation coefficients for $v c$-pairs for which a reduced system has non-zero transfer impedance. This is the case when a transmission path exists between two $v c$ nodes that do not pass by other $v c$-nodes.

A critical subject to performance evaluation of the algorithm is the scaling of iterations count with system size. No assessment of this scaling has been made at present.

With the choice of a Thevenin equivalent based system model the convergence properties include interesting considerations about stability of the physical system. The developed method is non-convergent if for instance active power injection at a node is higher than the crest of the $P-\delta$ curve. This is in [11] identified as a case of rotor angle instability. Exceptions to non-convergent cases may thus be implemented together with assessment of rotor angle stability.

The post-contingency Thevenin equivalents that are obtained together with PMU snapshots should be considered an asset of the method. It allows immediate association of contingency assessments with methods as discussed in [17] and [18] where a particular type of instability is detected on basis of Thevenin equivalents. 
By embracing the deficiencies represented by the system simplifications made with this study it is likely that the Thevenin equivalent based contingency analysis can indeed be a very powerful tool in real-time static security assessment. In particular, the ability of reproducing post-contingency nodal voltages obtained from time domain simulations and the general applications of system representation by Thevenin equivalents in post-contingency scenarios are interesting contributions of the method.

\section{CONCLUSION}

Ongoing developments in generation, operation paradigms and observability of power systems motivate development of fast and precise methods of assessing steady state post-contingency nodal voltages. Such a method is introduced and discussed. It is based on Thevenin equivalents referred in a common frame by grid transformation coefficients. Central assumptions include constant impedance loads and generation units with identical dynamic characteristics.

A small study is conducted to evaluate the developed method in comparison to Newton-Raphson's power flow method. It is found that the method reproduces steady state nodal voltages obtained from time domain simulations to a much better precision than Newton-Raphson's method. It is argued that a great asset of the method is the ability of obtaining post-contingency Thevenin equivalents for every voltage controlled node together with a coherent set of steady-state post-contingency nodal voltages. It is on this basis found likely that the Thevenin equivalent based method for static contingency assessment can evolve to be a powerful tool in security assessment of power systems.

\section{REFERENCES}

[1] Union of Coordination of Electricity Transmission (UCTE), "Fina Report of the Investigation Committee on the 28 September 2003 Blackout in Italy," April 2004.

[2] A. Phadke and R. de Moraes, "The wide world of wide-area measurements," IEEE Power and Energy Magazine, vol. 6, no. 5, pp. 52-65, September, 2008.

[3] M. Begovic, D. Novosel, D. Karlsson, C. Henville, and G. Michel, "Wide-area protection and emergeny control," Proceedings of the IEEE, vol. 93, No. 5, May 2005.

[4] H. Jóhannsson, A. Nielsen, and J. Østergaard, "Identification of critical transmission limits in injection impedance plane," International Journal of Electrical Power and Energy, vol. 43, no. 1, 2012.

[5] H. Johannsson, A. H. Nielsen, and J. Østergaard, "Wide-area assessment of aperiodic small signal rotor angle stability in real-time," IEEE Transactions on Power Systems, vol.28, no.4, pp.4545-4557, Nov. 2013.

[6] T. Weckesser, H. Jóhannsson, S. Sommer, and J. Østergaard, "Investigation of the adaptability of transient stability assessment methods to real-time operation," 3rd IEEE PES International Conference and Exhibition on Innovative Smart Grid Technologies (ISGT Europe), October 2012.

[7] A. Perez, H. Jóhannsson, P. Vancraeyveld, and J. Østergaard, "Suitability of voltage stability study methods for real-time assessment," 4th IEEE PES International Conference and Exhibition on Innovative Smart Grid Technologies Europe (ISGT EUROPE), October 2013.

[8] A. J. Wood, B. F. Wollenberg, Power System Generation Operation and Control. 2nd ed. John Wiley and sons, 1996.
[9] J. A. Huang, L. Loud, G. Vanier, B. Lambert, and S. Guillon, "Experiences and challenges in contingency analysis at Hydro-Quebec," IEEE Power and Energy Society General Meeting, July 2012.

[10] Y. Chen, Z. Huang, Y. Liu, J. M. Rice, and S. Jin, "Computational challenges for power system operation," 45th Hawaii International Conference on System Sciences 2012.

[11] P. Kundur, Power System Stability and Control. 2nd ed. Mc. GrawHill, 1994.

[12] S. H. Sommer and H. Jóhannsson, "Real-time Thevenin impedance computation,” 2013 IEEE PES Innovative Smart Grid Technologies. IEEE, 2013.

[13] E. Dmitrova, H. Jóhannsson, and A. H. Nielsen, "Assessment of the impact that individual voltage source has on a generator's stability," Proceedings of 10th International Power and Energy Conference, IPEC 2012. 2012.

[14] CIGRÈ TF38.02.08, "Long Term Dynamics Phase II, Final Report," March, 1993.

[15] IEEE Standards Association, Standard for Synchrophasor Measurements for Power Systems - IEEE Std C37.118.1-2011. December, 2011.

[16] O. Alsaç, B. Stott, and W. F. Tinney, "Sparsity-oriented compensation methods for modified network solutions," IEEE Transactions on Power Apparatus and Systems, Vol. PAS-102, No. 5, May 1983.

[17] T. Weckesser, H. Jóhannsson, J. Østergaard, and T. Cutsem, "Sensitivity based assessment of transient voltage sags caused by rotor swings," 18th Power Systems Computation Conference, Wroclaw, Poland, August 2014. Accepted.

[18] T. Weckesser, H. Jóhannsson, J. Østergaard, and T. Cutsem, "Early prediction of transient voltage sags caused by rotor swings," 2014 IEEE PES General Meeting, Washington, DC, July 2014. Accepted.

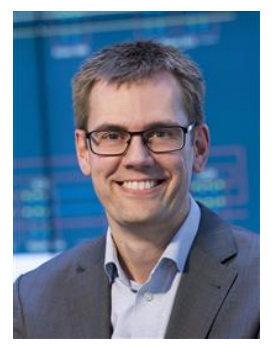

\section{BIOGRAPHIES}

Jacob Østergaard (SM'10) is Professor and Head of the Centre for Electric power and Energy, in the Department of Electrical Engineering, Technical University of Denmark. His research interests include integration of renewable energy, control architecture for future power system, and demand side. Professor Østergaard is serving in several professional organizations including the EU SmartGrids advisory council.

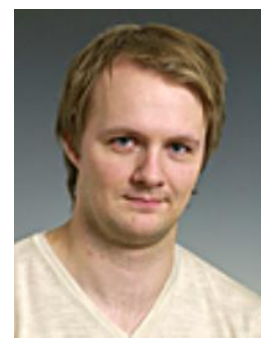

Hjörtur Jóhannsson (M'10) received the M.Sc. and the Ph.D. degree in Electrical Engineering from Technical University of Denmark, in 2007 and 2011 respectively. From 2011 to 2014 he was an assistant professor at CEE, Department of Electrical Engineering, Technical University of Denmark. From 2014 he continues his work for CEE as Senior Scientific Consultant. His research interests concern the development of methods that provide an early warning for stability and security problems in electric power systems.

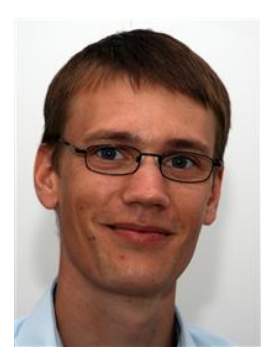

Jakob Glarbo Møller (M'13) received the M.Sc. degree in Electrical Engineering from Technical University of Denmark, in 2013. He is currently pursuing a Ph.D. at CEE, Department of Electrical Engineering, Technical University of Denmark. His research interests include methods for secure operation of sustainable power systems. 